\title{
Magnetic resonance imaging evaluation of intervertebral test spacers: an experimental comparison of magnesium versus titanium and carbon fiber reinforced polymers as biomateria?
}

\author{
T. Ernstberger · G. Buchhorn · G. Heidrich
}

Received: 21 March 2008/Accepted: 22 June 2009/Published online: 20 August 2009

(C) Royal Academy of Medicine in Ireland 2009

\begin{abstract}
Introduction Intervertebral spacers are made of different materials, which can affect the postfusion magnetic resonance imaging (MRI) scans. Susceptibility artifacts, especially for metallic implants, can decrease the image quality. This study aimed to determine whether magnesium as a lightweight and biocompatible metal is suitable as a bi material for spinal implants based on its MRI artifactin behavior.

Materials and methods To compare artifacting bo vis, we implanted into one porcine cadaveric srine dif $n$ ? test spacers made of magnesium, titanium a $a_{1}$ FFRP. All test spacers were scanned using tw T1-1 $>$ MRI sequences. The artifact dimensions w re traced on all scans and statistically analyzed.

Results The total artifact volume artifact area of the titanium spacers we tistically significantly larger than magnesium spacers $(F<0.001)$, while magnesium and CFRP sparers roduced almost identical artifacting behaviors $(P, 0$

Conclusion Orc resul syggest that spinal implants made with marno $\mathrm{m}$ alloys will behave more like CFRP devices in MRI scan.

T. Frns rger (k

D rtm Orthopaedic Surgery, University of Göttingen,

Rob Koch-Straße 40, 37075 Göttingen, Germany

e-mail: Astberger@med.uni-goettingen.de

G. Buchhorn

Biomaterial Laboratory, Department of Orthopaedic Surgery, University of Göttingen, Göttingen, Germany

G. Heidrich

Department of Diagnostic Radiology, University of Göttingen, Göttingen, Germany
\end{abstract}

Keywords Ma nom alloys - Innovative biomaterials . Interbody tesi plants - MRI artifacting

\section{Introduction}

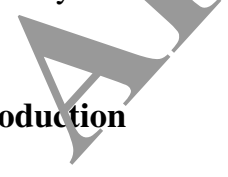

nal fusion devices such as implantable interbody spacer: are well-established and routinely used by spine surcons to keep adjacent vertebrae spaced apart while bone ingrowth and fusion take place. Such spacers also provide weight-bearing support between adjacent vertebrae. The principal state-of-the-art spinal implants are made from titanium alloys and carbon fiber-reinforced polymers (CFRP). These biomaterials have enjoyed clinical success and rapid widespread use by improving patient outcomes.

However, these materials have clinical and radiological limitations. Titanium is an excellently bioinert material that exhibits high biocompatibility. Titanium spacers produce good bone ingrowth without bone grafting. However, in magnetic resonance imaging (MRI) studies, titanium-based implants tend to cause distortion of the magnetic field which may obscure normal regional anatomy [1]. These properties pose difficulties in the postoperative MRI follow-up and evaluation of the fusion process due to the artifacting of its causes [5, 6].

The other principal material used for spacers consists of CFRP. Spacers made of this non-metallic biomaterial are not associated with the postoperative diagnostic problems of titanium because carbon produces a very low rate of artifact reactions and its radiolucency properties allow easier evaluation of the fusion process by MRI [3]. Carbon's modulus of elasticity affords good load-bearing with sufficient hardness. But unlike titanium, carbon spacers undergo poor osteointegration because a soft tissue interface develops around the material surface that prevents 
direct ingrowth of bone. As a result, carbon spacers have to be filled with bone allografts to achieve long-term stability $[1,3]$. CFRP implants have, therefore, been reviewed very critically in the literature [13].

Surgeons, over a century ago, recognized the potential of the lightweight metal magnesium as a biocompatible, osteoconductive, degradable implant material [7]. In 1907, Lambotte [7] was the first to introduce magnesium-based orthopedic devices; using a pure magnesium plate; he secured a bone fracture of the lower leg with gold-plated nails. A half a century later, magnesium-based metals were reported to have osteoconductive bioactivity and produce a more rapid formation of hard callus when used to support fractures in humans $[16,21]$. The large amounts of evidence supporting the clinical advantages of magnesium have been summarized in a recent review paper [14]. None of the studies to date have yet investigated the diagnostic behavior of magnesium in MRI. This situation motivated us to determine whether magnesium is a suitable biomaterial for spinal implants by studying its MRI artifacting behavior.

\section{Materials and methods}

To evaluate the behavior of spacers made with a magnesiu alloy, we compared their artifacting in diagnostic Mk. scans with that of spacers made of a conventional tanium alloy and of CFRP. We consecutively implant taree spacers made of each of the three biomaterials dimens in small, medium, and large sizes in one cad iv spine of a Gottingen mini pig (Figs. 1a-c, 2). The three $\mathrm{s}_{1}$ cers in group I were made of a magnesium-a iminium-manganese alloy (MgAlMn50), the three in gr $\mathrm{v}$ II of a titaniumaluminum-vanadium alloy (TiAl6V4), cnose in group III of a carbon fiber-reinforced 1 ner (CFRP).

Table 1 presents the implant characeristics. A cylinder was chosen as for spoce hape hecause cylinders have demonstrated lowest r. artifacting behavior [4]. The spacer sizesma medium, and large-were dimensioned the $\mathrm{s}$ for each group (height in $\mathrm{cm} \times$ base area in $\mathrm{cm}^{2}$. and th implant volume (IV) in $\mathrm{cm}^{3}$ and cross sect onal area (CSA) in $\mathrm{cm}^{2}$ was calculated for each size (Tabl Th spacer sizes were dimensioned as listed afte Nem an-Keuls multiple comparison analysis s' ved the selected sizes would produce significantly diffo $t$ artifacting behaviors $(P<0.001)$. Thus, a total of nine in 4 vidual spacers were implanted, scanned by MRI, and evaluated for their artifacting behavior on the scans.

Spacer implantation

For each serial MRI study, the cylindrical implant was placed exactly between two adjacent vertebrae of the

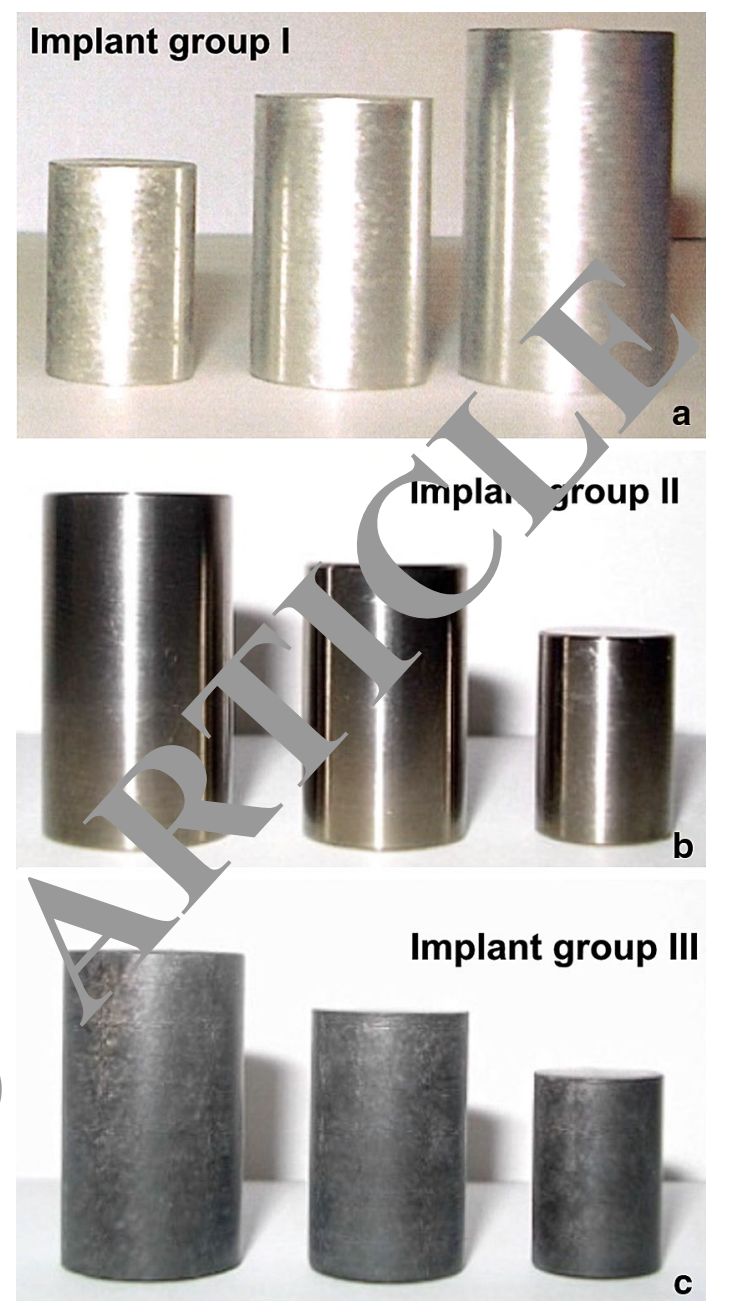

Fig. 1 Cylindrical test implants. a Magnesium (implant group I), b titanium (implant group II), c CFRP (implant group III)

cadaveric porcine spine. The spine with implant was then completely packed in a soft-tissue mass and placed in a plastic container [4]. To create comparable trial conditions, markings were drawn on the container wall to demarcate the vertebrae and implant positions. These demarcations were used to define the median artifact area (MAA). The container with the spine implanted with each spacer was examined by serial MRI (Fig. 3).

\section{Magnetic resonance imaging}

Magnetic resonance imaging was performed with a $1.5 \mathrm{~T}$ MRI (Magnetom Symphony, Siemens AG Medical Solutions, Erlangen, Germany). The T1w-TSE sequences were used to acquire a slice thickness of $3 \mathrm{~mm}$ (Fig. 3a-c) which included a first sequence (TR 600; TE 14; flip angle 15; band width 150), and a second sequence (TR 2,260, TE 14, flip angle 15, band width 150). We selected a matrix of $512 \times 512$ pixels combined with a field of view (FOV) of $500 \mathrm{~mm}$. The T1w-TSE sequence has been established to 


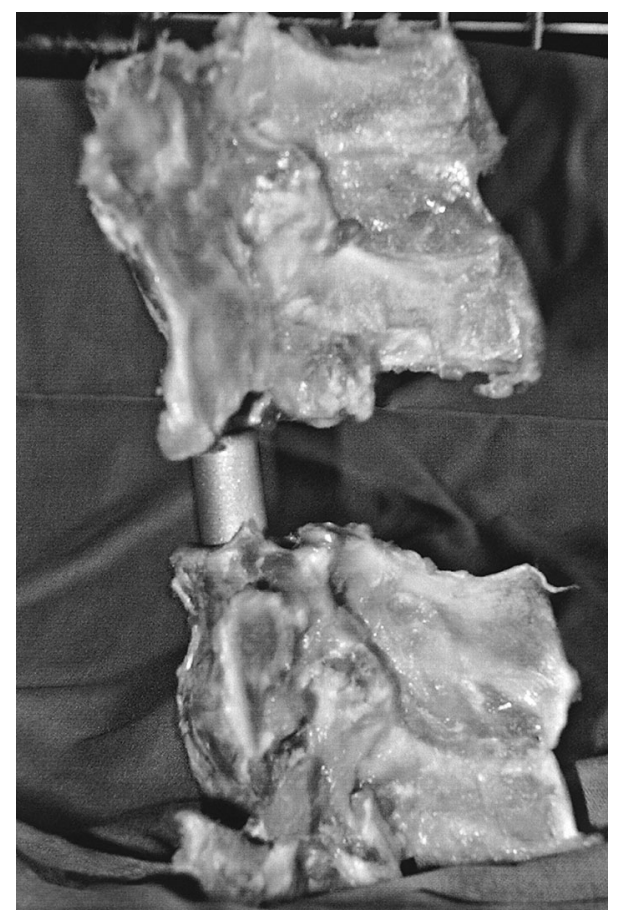

Fig. 2 Cadavaric porcine spine model with an implanted medium titanium test cylinder produce best imaging results for implants and the least amount of intrinsic artifacting [3, 5, 6, 8-10, 12, 20].

Using a current version of DICOM reader software, one author (TE) measured the artifact area on the scan of each of the nine implants six times, i.e., a total of 54 individual tracings were recorded and analyzed. The measurements started with the slice with the first artifacting reaction and ended with the last slice exhibiting an ifa rèction. Corresponding to the respective implant's $\mathrm{CS}$. the middle slice of all slices exhibiting artifact reactions was defined as the MAA for each implant. Tocalc te th total artifact volume (TAV) for each space, all artifa areas measured for that spacer were added nd mulliplied by the slice thickness of $3 \mathrm{~mm}$ acc din the multisection slice technique described b, De, in et al. [2]. The ratio of CSA to MAA and the r of IV TAV were calculated and presented in tabls ( 1 . 1 ).

Statistical

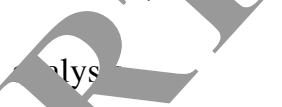

Newm-Keuls aultiple comparisons were used to calculate intr o a differences in TAV and MAA (Table 2). $T$ test correlations were performed to determine any intergroup differences regarding the implant materials ble 2). A $P$ value of $<0.05$ indicated a significant differ nce between the means of any two groups.

Table 1 Spacer dimensions

\begin{tabular}{llll}
\hline $\begin{array}{l}\text { Sizes for } \\
\text { all groups }\end{array}$ & $\begin{array}{l}\text { Dimensions } \\
\text { Height } \times \text { base } \\
\text { area }\left(\mathrm{cm} \times \mathrm{cm}^{2}\right)\end{array}$ & $\begin{array}{c}\text { Cross sectional } \\
\text { area }\left(\mathrm{CSA}, \mathrm{m}_{1}\right.\end{array}$ & $\begin{array}{l}\text { Imp. } \\
\text { volume } \\
\left.\mathrm{TV}, \mathrm{cm}^{3}\right)\end{array}$ \\
\hline Small & $1.5 \times 0.78$ & 1.5 & 1.2 \\
Medium & $2.0 \times 1.13$ & 2.4 & 2.3 \\
Large & $2.5 \times 1.54$ & 28 & 3.5 \\
\hline
\end{tabular}

\section{Results}

Table 1 presents the spacer dimensions. Table 2 shows the intragroup comparisons of target variables. Table 3 lists the results of the intergroup $t$ test correlations between TAV and MAA in relation to spacer material. Mean artifacting behavior increased with spacer size. When magnesium was
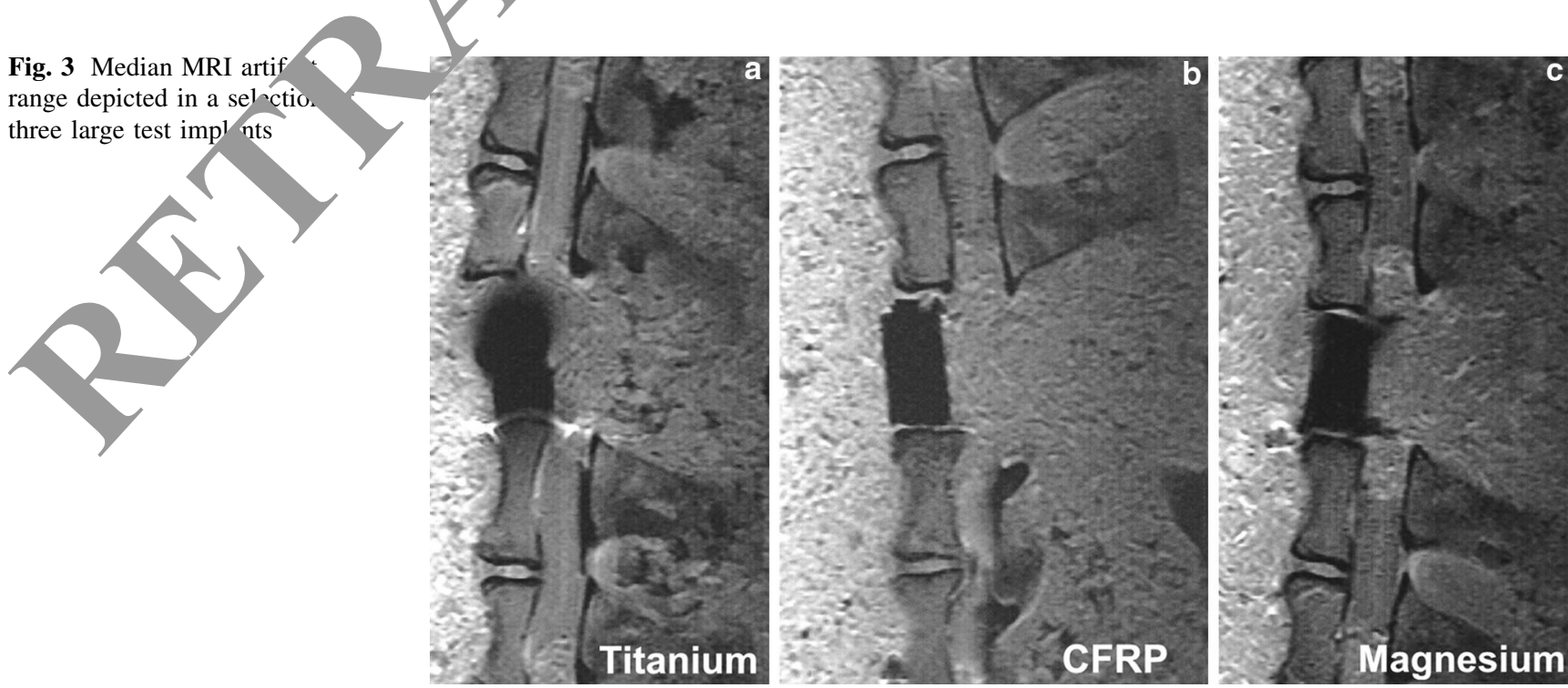
Table 2 Intragroup comparison of target variables

\begin{tabular}{|c|c|c|c|c|c|}
\hline Spacer material & Size & $\begin{array}{l}\mathrm{MAA}^{\mathrm{a}} \mathrm{cm}^{2} \\
\text { Mean } \pm \mathrm{SD}\end{array}$ & $\begin{array}{l}\text { Ratio } \\
\text { CSA:MAA }\end{array}$ & $\begin{array}{l}\mathrm{TAV}^{\mathrm{a}} \mathrm{cm}^{2} \\
\text { Mean } \pm \mathrm{SD}\end{array}$ & $\begin{array}{l}\text { Ratio } \\
\text { IV:TAV }\end{array}$ \\
\hline \multirow[t]{3}{*}{ Group I MgAlMn50 $(n=3)$} & Small & $1.91 \pm 0.04$ & $1: 1.3$ & $1.83 \pm 0.09$ & $1: 1.5$ \\
\hline & Medium & $3.26 \pm 0.06$ & $1: 1.4$ & $4.17 \pm 0.09$ & $1: 1.8$ \\
\hline & Large & $4.06 \pm 0.07$ & $1: 1.2$ & $5.08 \pm 0.15$ & $1: 1.3$ \\
\hline \multirow[t]{3}{*}{ Group II TiAl6V4 $(n=3)$} & Small & $3.26 \pm 0.04$ & $1: 2.2$ & $5.71 \pm$ & $1: 4.8$ \\
\hline & Medium & $4.61 \pm 0.23$ & $1: 1.9$ & $9.32 \pm 0.10$ & 1:4.1 \\
\hline & Large & $5.54 \pm 0.04$ & $1: 1.6$ & 4 & $1: 2.9$ \\
\hline \multirow[t]{3}{*}{ Group III CFRP $(n=3)$} & Small & $1.89 \pm 0.07$ & $1: 1.3$ & & $1: 1.5$ \\
\hline & Medium & $3.18 \pm 0.06$ & $1: 1.3$ & 1008 & $1: 1.7$ \\
\hline & Large & $4.06 \pm 0.13$ & $1: 1.2$ & $5.08 \pm 013$ & $1: 1.3$ \\
\hline
\end{tabular}

Table 3 Intergroup comparisons of artifacting behavior by $t$ test correlation

\begin{tabular}{llcr}
\hline Spacer material & Size & \multicolumn{2}{l}{$P$ value $^{\mathrm{a}}$} \\
\cline { 3 - 4 } & & MAA & TAV \\
\hline Group I versus group II & Small & $\leq 0.001$ & $\leq 0.00$ \\
& Medium & $\leq 0.001$ & $\leq 0.00$ \\
& Large & $\leq 0.001$ & $\leq 0.00$ \\
Group I versus group III & Small & 0.59 & 0.0 . \\
& Medium & 0.09 & 0.26 \\
& Large & 1.0 & $0 \%$ \\
\hline
\end{tabular}

$M A A$ median artifact area, $T A V$ total artifact volume

${ }^{a}$ Significance level $P<0.05$

compared with titanium, there were gnificant differences in both MAA and TAV. When magi ium was compared with carbon, the differences were not s.outicant. In fact, magnesium produces an artifact to nuior very similar to that of CFRP.

\section{Discussion}

Spinal surg _ons have ot stopped searching for the optimum $\mathrm{sp}^{\mathrm{r}}$ - naterial that combines high biocompatibility with artifa free MRI imaging behavior in the implant enyro hent. ais study conducted to determine whether $\mathrm{G}_{\mathrm{dr}}$ acers made of the biomaterial magnesium are suita as spinal implants by comparing their MRI artifacting with that of identically dimensioned spacers made of a titanium alloy and a carbon fiber-reinforced polymer.

In radiological spinal diagnostics, MRI is highly effective for clarifying postfusion questions regarding osseus and soft-tissue structures in relation to implant position. A comparative in vitro study shows that MRI has a higher sensitivity than CT in recting osseus changes in the implant's d ect arroundings [19]. Moreover, MRI is well suited to a mstrace myelopathies, inflammatory and infectious proce. $s$, and any neurodegenerative changes. The Mu ing behavior of spinal implants is obviously well doc mented in the literature [8, 10-12, 15, 17, 18, 20]. However, the aims of the published studies differed in that st focused on determining sequence-related artifact size. In phantom study by Rudisch et al. [11], the relevance of metallic artifacts and implant-related characteristics, such as implant material and position, was demonstrated in addition to effects caused by the selected MRI sequence. In materials with a higher magnetizability like titanium alloys, implant shape additionally has an effect on the range of MRI artifacts [4].

The results of this comparative study showed that implant material and volume both affected the MRI artifacting behavior of our cylindrical test spacers. It was also noted that the smaller the implant size, the smaller was the range of susceptibility artifacts produced. The ratios calculated in Table 2 prove that the magnesium metal alloy exhibited behavior artifacting that was more like a non-metal.

Our results confirm previous findings that MRI artifacting caused by solid implants is influenced by implant material, volume, and shape [4]. Judging from its nonmetal-like MRI artifacting behavior alone, magnesium would appear to be a more suitable biomaterial for spinal implants than titanium. Given its osseoconductive potential as a metal [7], implant alloys made with magnesium would combine the advantages to the two principal spacer materials currently used, but without their limitations, at least in terms of MRI artifacting. Hence, magnesium alloys may show promise as spinal implants.

Acknowledgments The authors thank Dr. Mark Riner of MedTech Composites GmbH, Switzerland, the Peter Brehm Company, 
Weisendorf, Germany, and the Material Science at the Technical University of Hannover, Germany for producing the respective test implants.

\section{References}

1. Brantigan JW, Steffee AD (1993) A carbon fiber implant to aid interbody lumbar fusion. Two-year clinical results in the first 26 patients. Spine 18:2106-2107

2. Debatin JF, Nadel SN, Sostman HD, Spritzer CE, Evans AJ, Grist TM (1992) Magnetic resonance imaging-cardiac ejection fraction measurements. Phantom study comparing four different methods. Invest Radiol 27:198-204. doi:10.1097/00004424-19920300000003

3. Ernstberger T, Heidrich G, Bruening T, Krefft S, Buchhorn G, Klinger HM (2006) The interobserver-validated relevance of intervertebral spacer materials in MRI artifacting. Eur Spine $\mathbf{J}$ $7: 1-7$

4. Ernstberger T, Heidrich G, Buchhorn G (2007) Post implantation MRI with cylindric and cubic intervertebral test implants: evaluation of implant shape, material and volume in MRI artifactingan in vitro study. Spine J 7:353-359. doi:10.1016/j.spinee. 2006.03.016

5. Henk CB, Brodner W, Grampp S, Breitenseher M, Thurnher M, Mostbeck GH, Imhof H (1999) The postoperative spine. Top Magn Reson Imaging 10:247-264. doi:10.1097/00002142199908000-00006

6. Herold T, Caro WC, Heers G, Perlick L, Grifka J, Feuerbach S, Nitz W, Lenhart M (2004) Influence of sequence type on the extent of the susceptibility artifact in MRI-a shoulder specin. study after suture anchor repair. Rofo 176:1296-1301

7. Lambotte A (1932) L'utilisation du magnesium comme materiel perdu dans l'ostheosynthèse. Bull Mem Coc Nat Chii 1 :51334

8. Malik AS, Boyko O, Atkar N, Young WF (2001 compara study of MR imaging profile of titanium pe licle ews. Acta Radiol 42:291-293. doi:10.1080/02841850 2734684

9. Ortiz O, Pait TG, McAllister P, Sauter K (1996) Postoperative magnetic resonance imaging with titar $m$ implal ts of the thoracic and lumbar spine. Neurosur, 38\%41-745. doi: 10.1097/00006123-199604000-00022

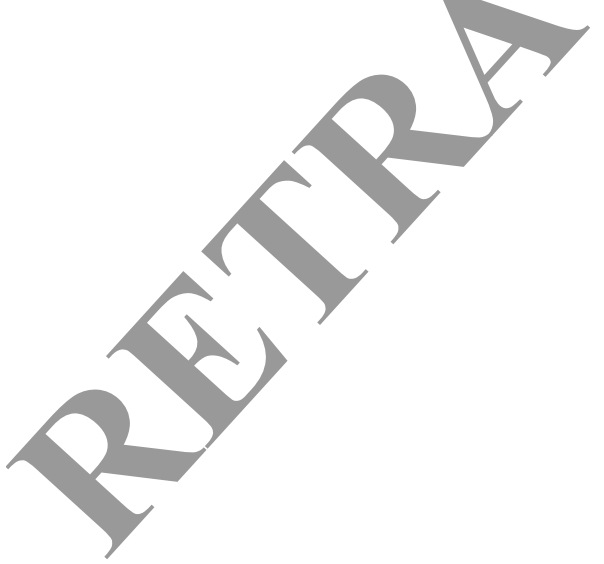

10. Petersilge CA, Lewin JS, Duerk JL, Yoo JU, Ghaneyem AJ (1996) Optimizing imaging parameters for MR evaluation of the spine with titanium pedicle screws. Am J Roentenol 166:1213-1218

11. Rudisch A, Kremser C, Peer S, Kathrein A, Judmaier W, Daniaux $H$ (1998) Metallic artifacts in magnetic resonance imaging of patients with spinal fusion. A comparison of implant materials and implant sequences. Spine 23:692-699. doi:10.1097/ 00007632-199803150-00009

12. Rupp R, Ebraheim NA, Savolaine ER, Jarkson WT (1993) Magnetic resonance imaging evaluation of $\mathrm{m}_{\mathrm{m}} \mathrm{vi}$. . wi w metal implants. General safety and superior imagins ith itanium. Spine 18:379-385

13. Schreiner U, Schwarz M, Scheller G, roeder- oersch H, Jani L (2000) Knöchernes Einwachsve alte. n Probekörpern aus kohlefaserverstärktem Kunsts off. Z Ort $\mathrm{p}$ Ihre Grenzgeb 138:540-543. doi:10.1055/s-2 0-9598

14. Staiger MP, Pietak AM, Yuad J, Di ss G (2006) Magnesium and its alloys as orthopae bionmenals: a review. Biomaterials 27:1728-1734. doi:10.1016/J materials.2005.10.003

15. Thomsen M, Sch 'er U, Bre och SJ, Hansmann J, Freund M (2001) Artefac an vromagnetism dependent on different metal alloys magnet esonance imaging. An experimental study. Ort'iopac 30:540-544. doi:10.1007/s001320170063

16. Troitskin DN (1944) The resorbing metallic alloy 'Ostheosinth. as material for fastening broken bone. Khirurgiia $8: 4^{-14}$

17. Vaciary Chesnut RM, Scuderi G, Healy JF, Massie JB, Garfil so (1994) Metallic spinal artifacts in magnetic resonance imagin Spine 19:1237-1242

8. Van Goethem JW, Parizel PM, Jinkins JR (2002) Review article: MRI of the postoperative lumbar spine. Neuroradiology 44:723739. doi: $10.1007 / \mathrm{s} 00234-002-0790-2$

19. Walde TA, Weiland DE, Leung SB, Sychterz CJ, Ho S, Engh CA, Potter HG (2005) Comparison of CT, MRI and radiographs in assessing pelvic osteolysis: a cadaveric study. Clin Orthop Relat Res 437:138-144. doi:10.1097/01.blo.0000164028.14504.46

20. Wang JC, Sandhu HS, Yu MD, Minchew JT, Delamarter RB (1997) MR parameters for imaging titanium spinal instrumentation. J Spinal Disord 10:27-32. doi:10.1097/00002517-1997 02000-00004

21. Znamenskii MS (1945) Metallic ostheosynthesis by means of an apparatus made of resorbing metal. Khirurgiia 12:60-63 